\title{
A Markov Based Detection for Broadband over Power Line Communications (PLC)
}

\author{
M. Morshed ${ }^{a^{*}}$, P. Sarker ${ }^{a^{*}}$, R. C. Roy ${ }^{b}$ \\ ${ }^{a}$ Department of Electrical and Electronic Engineering, Ahsanullah University of Science and Technology, Dhaka, \\ and ${ }^{b}$ Pilot Plant and Process Development Center (PP \& PDC), BCSIR, Dhaka, Bangladesh
}

\begin{abstract}
Successful development of Broadband over Power Lines (BPL) is obviously a potential solution for wireless communication systems with the existing infrastructure. Multi-Carrier Code Division Multiple Access (MC-CDMA) with Orthogonal Frequency Division Multiplexing (OFDM) modulation technique is a promising solution for a frequency selective fading with impulsive noises of the PLC channel. A linear iterative receiver with a Finite State Markov Channel (FSMC) model is proposed to remove Multiple Access Interference (MAI) and Inter Symbol Interferences (ISI). In this paper we focused on Maximum Likelihood (ML) feedback detection by introducing FSMC to get significant improvement in Bit Error Rate (BER). This model of the receiver is more robust against Gaussian colored and impulsive noises of the PLC channel.
\end{abstract}

Keywords: FSMC, MC-CDMA, OFDM, MAI, ISI, etc.

\section{Introduction}

Power Lines have been designed to carry alternating current (AC) (50 or $60 \mathrm{~Hz}$ ) for distribution of electrical energy through wiring in houses or buildings. Some coupling radio frequency devices have operated below $2 \mathrm{MHz}$ with limited communications capabilities. It has been difficult to achieve dependable high-speed communications due to power line characteristics. For remote customers, where fiber and cable backbone systems are not economical, PLC is still a good cost-effective service though reliable high speed data transmission is a great challenge. The presence of the noisy loads in the transmission network, colored noises and disturbances produced outside the transmission line, like switching, corona, impulse noises etc., result high level interference signals (Meng H. et al. 2005, Zimmermann M. et al. Feb, 2002). The PLC signal is mainly influenced by three main factorsimpedance, attenuation and Signal-to-Noise Ratio (SNR) (Hasan M.M. et al. 2006). Considering all these aspects, high-frequency power-line characteristics become undulated with increase of BER in the high bit-rate data transmission.

Due to the robustness of the application of Orthogonal Frequency Division Multiplexing (OFDM) for power line communication, it is suitable against impulsive noise, multipath and frequency selective fading. Moreover, OFDM uses bandwidth more efficiently and is easy for spectrum shaping. (Aghajeri S. et al. 2002). Homeplug ${ }^{\circledR}$ AV has chosen as a standard for broadband over power line, which uses OFDM
(HomePlug ${ }^{\circledR}$ AV White Paper, HomePlug ${ }^{\circledR}$ Powerline Alliance, Inc. 2005). A transceiver with OFDM and MCCDMA technique with an iterative receiver is proposed in (Le Nir V. et al. 2006), considering colored noise. In our paper we proposed an iterative receiver with a Finite State Markov Channel (FSMC) model to correct feedback errors.

MC-CDMA is a spread spectrum signaling system with advantageous robustness against multipath and multiuser interferences. For the downlink of broadband cellular systems, MC-CDMA scheme is already a proven access technique because of its narrow-band interference rejection, high spectral efficiency, robustness in frequency selective channels with a low complexity at receiver, multiple access capability with high flexibility, efficient utilization of the available bandwidth, allowing high data rates (Hara S. et al. 1997). To transmit $N$ symbols simultaneously in an OFDM system, the available bandwidth $W$ is divided into $N$ subchannels (subcarriers). The orthogonality between the subcarriers is maintained by adding a cyclic prefix to the transmitted vector. The length of the cyclic prefix has to be as large as the length of the Channel Impulse Response (CIR). Each of the subchannels experiences flat fading if the length of the cyclic prefix is greater than or equal to the length of the CIR, even if the original channel is frequency selective.

* Corresponding authors: E-mail address: monjurm@aust.edu; palashsarker.eee@aust.edu 


\section{PLC Transmission System}

The complex symbols $\left\{S_{k}\right\}_{k=0}^{N-1}$ are transmitted by OFDM modulator. The OFDM modulated signal can be expressed as

$$
s(t)=\sum_{k=0}^{N-1} S_{k} e^{j 2 \pi f_{k} t} \text { for } 0 \leq t \leq T_{s}
$$

where $f_{k}=f_{o}+k \Delta f . T_{s}$ and $\Delta f$ are called symbol duration and subchannel space of OFDM respectively. The symbol duration must be long enough such that $T_{S} \Delta f=1$. This is the condition for orthogonality for proper demodulation. The OFDM signal can be demodulated by -

$$
\frac{1}{T_{s}} \int_{0}^{T_{s}} s(t) e^{-j 2 \pi f_{k} t} d t
$$

Since Fast Fourier Transform (FFT) algorithm provides an efficient way to implement the Discrete Fourier Transform (DFT) and the Inverse Discrete Fourier Transform (IDFT), it reduces the number of complex multiplications from $N^{2}$ to $-\frac{\mathrm{N}}{2} \log _{2} N$ for $N$ points. Therefore, the OFDM transmitter and receiver can be implemented using the IDFT and DFT respectively. The MC-CDMA transmitter consists of a channel coder, bit interleaver, spreading module, symbol interleaver and an OFDM modulator as proposed proposed in (Le Nir V. et al. 2006). The proposed receiver on the other hand has gotten the reverse process with a linear Minimum Mean Square Error (MMSE) equalizer, a Soft Output Viterbi Algorithm (SOVA) with a FSMC model for reliable decoding.

The spread coded vector at first is applied to symbol interleaver and an OFDM modulator. The modulation is performed by Inverse Fast Fourier Transform (IFFT) with a guard interval insertion. This signal is transmitted through the PLC channel. The channel adds the noises mentioned in the introduction which is colored in nature. It is then passed through a whitening filter. The demodulation is done after removal of the guard interval. The received vector after whitening can be represented by -

$$
\mathrm{r}=\left[\mathrm{FHF}^{\mathrm{H}} \mathrm{S}+\underline{\mathrm{n}}\right] \frac{1}{\mathrm{Q}}
$$

where -

$\mathbf{F}$ and $\mathrm{F}^{\mathrm{H}}$ are FFT and IFFT operation

$\underline{\mathbf{H}}$ is the equivalent channel matrix

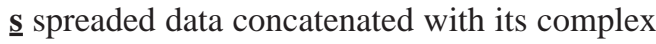
conjugate

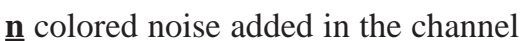

I/Q whitening filter

This received vector is then passed through an MMSE with equalization coefficient as given proposed in (Le Nir V. et al. 2006).

$$
g_{k}=\frac{\lambda_{k}^{*}}{\left|\lambda_{k}\right|^{2}+\frac{1}{\gamma}}
$$

where -

$\gamma$ is the Signal to Noise Ratio (SNR) of the received vector.

$\lambda_{\mathrm{k}}$ is a diagonal matrix.

Finally, estimated vector $\gtrless_{\circ}^{\circ}$ is found after deinterleaving and dispreading operation. It is then passed through a SoftOutput Viterbi Algorithm (SOVA) which computes the reliability of the information bits as a Log-Likelihood Ratio (LLR). The operation of a SOVA decoder can be divided into two parts. In the first part, decoding is done by selecting the most likely coded sequence. In the second part of SOVA decoding, the bits from the trellis are computed using backward traverse Viterbi algorithm, and the algorithm computes matrices and paths, starting at $i=N$ and ending at $i=0$

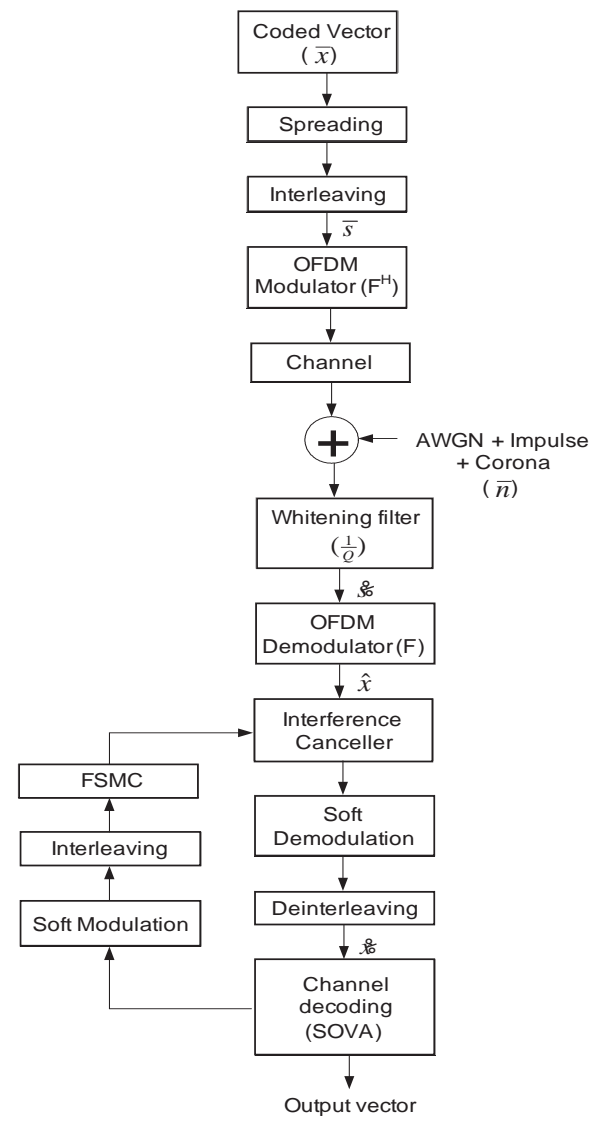

Fig. 1. Complete Block Diagram of PLC system. 
(Robert H. et al. 2006). With QPSK modulation the priori LLR probabilities of the coded bits can be approximated as:

$$
\Lambda\left(u_{i}\right)_{L L R}^{\operatorname{Pr} i}=\log \left(\frac{\operatorname{Pr}\left(u_{i}=-\left.1\right|^{\circ}\right)}{\operatorname{Pr}\left(u_{i}=+\left.1\right|^{\circ}\right)}\right) \approx \operatorname{Re}\left(x^{\circ}\right)+\operatorname{Im}\left(x^{\circ}\right)
$$

For re-estimation of the received symbols, posteriori LLRs are interleaved again. An $M$-ary conversion is done to feed into FSMC model. The probabilities of the state transitions occur to the adjacent states are given in (Ekpenyong A.E. et al. 2006).

$$
\begin{gathered}
\left.P_{i, j} \stackrel{\Delta}{=} \operatorname{Pr}\left(S(n)=S_{i} \mid S(n-1)=S_{j}\right)=0\right) \\
\quad \text { for }|\mathrm{i}-\mathrm{j}|>1
\end{gathered}
$$

where -

$S(n)$ is the state at time $n$

$\mathrm{P}_{\mathrm{i}, \mathrm{j}}$ is the state transition probability

Some state transitions are avoided for erroneous transmission with the following state-transition probabilities as in (Ekpenyong A.E. et al. 2006). The output of the FSMC model is then feedback to the Interference Canceller to reconstruct the transmitted signal. Normally four to six iterations are enough for optimum results.

\section{Channel Model}

Branching of multiple lines with different characteristic impedances and loads connected to the electrical network cause impedance mismatch. For this reason the signal is received in multiple reflected paths. Reflection occurs at the locations of impedance changes, like line terminals, in homogeneities and in faults. Therefore, it is reasonable to consider the channel as multipath channel (Zimmermann M. et al. Apr.2002). Any transmission scheme, related to power line channel is very noisy. Power line channel, having different types of noise sources- switching of power supply appliances, universal motors, short wave amateur radio transmission, lightening, network switching, impulse noise, corona noise etc. can be generated within or outside the channel. In frequency domain channel transfer function is described as in (Zimmermann M. et al. Apr.2002).

$$
H(f)=\sum_{i=1}^{N_{P}} g_{i} \cdot e^{-\left(a_{0}+a_{1} f^{k}\right) d_{i}} \cdot e^{-j 2 \pi f\left(d_{i} / v_{p}\right)}
$$

where -

$g_{i}$ is the weighting factor

$a_{o}, a_{1}$ are the attenuation factors

$k$ is the exponent of the attenuation factor

$N_{p}$ is the number of paths

$d_{i}$ is the length of these path

$v_{p}$ is the propagation velocity of the cable
Amongst all noises, corona is a common noise in high voltage power line which is dependent on the service voltage, the geometric configuration of the power line and the type of conductors involved in the line. Corona noise can be synthesized with the spectrum of an autoregressive digital filter whose output can be expressed as in (Haykin S. 2001). Two rigorous channel characteristics defined as good and bad channels are chosen from (Le Nir V. et al. 2006) for simulation which describe typical PLC transfer function.

\section{Simulations}

Simulations were carried out for $25 \mathrm{MHz}$ bandwidth with $50 \mathrm{MHz}$ sampling frequency. After QPSK mapping, half-rate convolutional encoder is used for channel coding. The coded vector is spread by a Fourier matrix of spreading length 2048. Then the data is bit-interleaved (2048). Simulation is done with 100 OFDM symbols. So the size of the symbol interleaver is 204800. The OFDM modulator is used with FFT size equal to $2 \times$ number of subcarriers. In our case 2048 subcarriers give an FFT size of 4096. The cyclic prefix of length 256 is added as guard band. For good channel with $v_{p}=0.5 c$, the maximum delay spread is $2 \mu$ s and that for the bad channel is $3.4 \mu$ s.

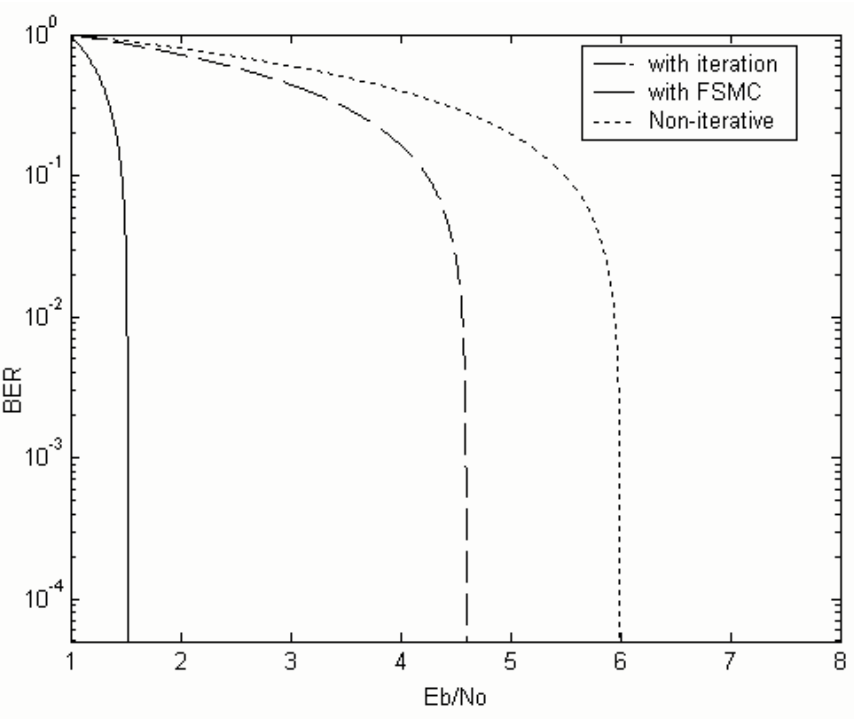

Fig. 2. Performance evaluation of QPSK Non-iterative and iterative MC-CDMA receiver on bad channel.

Fig. 2 shows the BER of the iterative MC-CDMA receiver with $10 \mathrm{~dB}$ impulsive colored noise. The proposed receiver can recover the data of the different users without the effect of noise. The corona has negligible effect. 
Fig. 3 shows the Bit Error Rate (BER) performance of a MCCDMA system with the parameters of good and bad channels whose parameters are given in (Langfeld P. et al. 2001). Integration of FSMC model in the iterative receiver makes the decoding almost independent of the channel parameters.

Fig. 4 shows the difference between the performance of the noniterative receiver and that of the iterative receiver with FSMC for good channel parameters only.

\section{Conclusion}

In this paper, an MC-CDMA transmission with iterative receiver is proposed for the PLC channel with OFDM modulation which is more robust against the noises in the existing power channel. It does not interfere with other systems such as Shortwave radio, UHF etc.

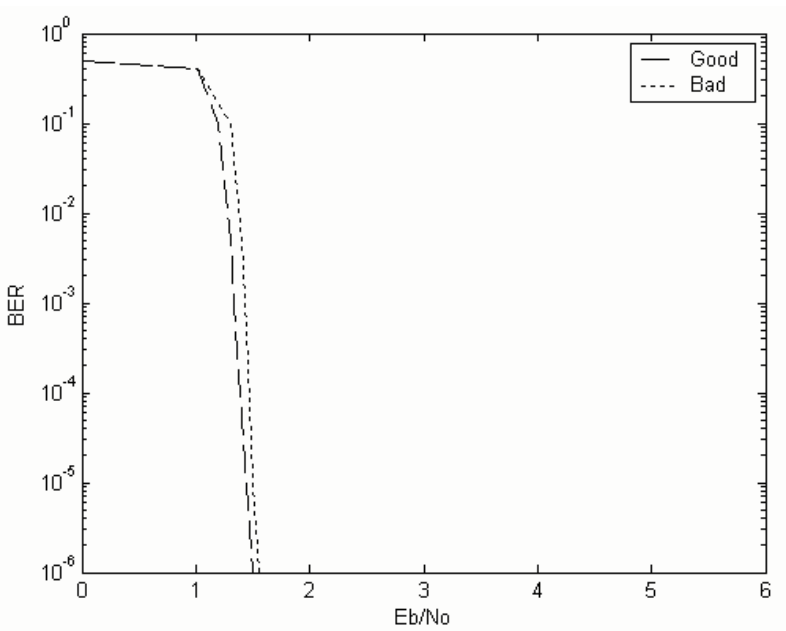

Fig. 3. Performance evaluation of QPSK iterative MC-CDMA receiver with good and bad channels.

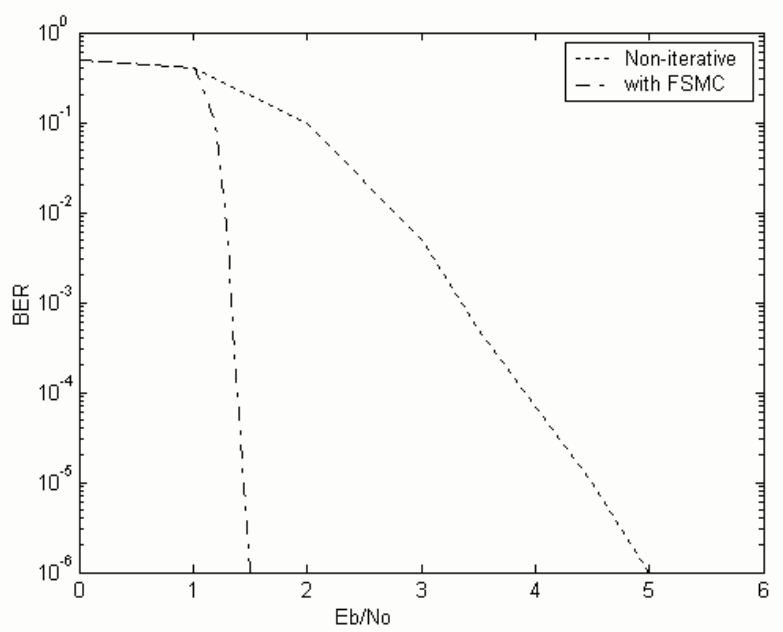

Fig. 4. Performance evaluation of QPSK Non-iterative and iterative MC-CDMA receiver on good channel.

\section{References}

Aghajeri S., Shafiee H. R. and Mohammadpour Velni J. (2002) Design of an OFDM System for High Rate Communication over Low Voltage Power Lines, Proceedings of the 10th Mediterranean Conference on Control and Automation MED2002 Lisbon, Portugal, July 9-12.

Ekpenyong A. E. and Huang Y. F. (Oct. 2006) Feedback-Detection strategies for adaptive modulation systems, IEEE trans. On Communications, 54(10): 1735-1740.

Hasan M. M., Matin M. A. and Rahman S. (December 2006) Channel behavior of residential powerline cable for the broadband PLC, 4th International Conference on Electrical and Computer Engineering ICECE 2006, 19-21 Dhaka, Bangladesh.

Hara S., Prasad R. (Dec. 1997) Overview of multicarrier CDMA, IEEE Communications Magazine, 35(12): 126-133.

Haykin S. (2001) Adaptive Filter Theory, Prentice-Hall, Englewood Cliffs, NJ, USA, 4th edition.

Meng H., Guan Y. L. and Chen S. (April 2005) Modeling and analysis of noise effects on broadband power-line communications,. IEEE Trans. Power Delivery, 20(2): 630-637.

Langfeld P. and Dostert K. (April,2001) The Capacity of typical Powerline Reference Channels and Strategies for System Design in Proc. ISPLC 2001, Malm, Sweden, pp. 271-278.

Le Nir V. and Moonen M. (2006) A MC-CDMA iterative solution for Broadband over Powerline Communications. 14th European Signal Processing Conference (EUSIPCO'2006), September 4-8, Florence, Italy.

HomePlug ${ }^{\circledR}$ AV White Paper, HomePlug ${ }^{\circledR}$ Powerline Alliance, Inc., 2005.

Pighi R. and R. Raheli (2007) Linear Predictive Detection for Power Line Communications Limited by Colored Noise. EURASIP Journal on Applied Signal Processing, special issue on, Advanced Signal Processing and Computational Intelligence Techniques for Power Line Communications.

Robert H. Morelos-Zaragoza (2006). The Art of Error Correcting Coding, Second Edition. John Wiley \& Sons Ltd. ISBN: 0470-01558-6

Zimmermann M. and Dostert K. (February 2002) Analysis and modeling of impulsive noise in broadband power-line communications. IEEE Trans. Electromagnetic Compatibility, 44(1): 249-258.

Zimmermann M. and K. Dostert (April 2002) A multipath model for the powerline channel. IEEE Trans. Commun., 50(4): 553-559.

Received : June 06, 2009;

Accepted : July 24, 2009 